\title{
A Mixed Covolume Method For The Pseudo-Parabolic Integro-Differential Equation On Triangular Grids
}

\author{
Xiaomei Zhang ${ }^{1, a} \quad$ Ziwen Jiang ${ }^{2, b}$ \\ ${ }^{1}$ Basic Courses Department, Shandong Yingcai College, Jinan, 250104, P. R. China \\ ${ }^{2}$ School of Mathematical Sciences, Shandong Normal University, Jinan, 250014, P. R. China \\ E-mail: ${ }^{a} z h x m 800717 @ 163 . c o m,{ }^{b}$ ziwenjiang@163.com
}

Keywords: Pseudo-parabolic integro-differential equation; mixed covolume methods;generalized mixed covolume elliptic projection; error estimates.

Abstract. In this paper, we present a mixed covolume method for the initial-boundary value problem of the pseudo-parabolic integro-differential equation. This method uses the lowest order

Raviart-Thomas mixed element space on triangles as the trial space. The convergence analysis shows that this method yields the approximate solution with optimal accuracy in $\mathbf{H}(\operatorname{div} ; \Omega) \times L^{2}(\Omega)$.

\section{Introduction}

Consider the following initial-boundary value problem of the pseudo-parabolic integrodifferential equation

$$
\begin{array}{lc}
\text { (a) } u_{t}=\operatorname{div}\left(a \nabla u_{t}+b_{1} \nabla u+\int_{0}^{t} b_{2} \nabla u d \tau\right)+f,(\mathbf{x}, t) \in \Omega \times(0, T], \\
\text { (b) } u(\mathbf{x}, t)=0, & (\mathbf{x}, t) \in \partial \Omega \times(0, T], \\
\text { (c) } u(\mathbf{x}, 0)=u_{0}(\mathbf{x}), & \mathbf{x} \in \Omega .
\end{array}
$$

where $\Omega$ is a bounded convex polygonal domain in $\mathrm{R}^{2}$ with the boundary $\partial \Omega, 0<T<\infty . u_{t}=\frac{\partial u}{\partial t}, \nabla$ and div denote the gradient and the divergence operators, respectively. The functions $a, b_{1}, b_{2}$ with their derivatives are smooth enough, and there exist two positive constants $c_{1}$ and $c_{2}$ such that $0<c_{1} \leq a \leq c_{2}$. Here and in what follows, we will not write the independent $\mathrm{x}, t, \tau$ for any functions unless it is necessary. Vectors will be expressed in boldface.

Introduce a new variable $\mathbf{p}=-\left(a \nabla u_{t}+b_{1} \nabla u+\int_{0}^{t} b_{2} \nabla u d \tau\right)$, and let $\alpha=a^{-1}, b=\alpha b_{1}, \beta=-\nabla b$, $c=\alpha b_{2}, \gamma=-\nabla c$, then (1.1) can be written as a system of first-order partial differential equations

$$
\begin{array}{ll}
\text { (a) } \left.a \mathbf{p}+\nabla u_{t}+\nabla(b u)+\beta u+\int_{0}^{t} \nabla(c u) d \tau\right)+\int_{0}^{t} \gamma u d \tau=0,(\mathbf{x}, t) \in \Omega \times(0, T], \\
\text { (b) } u_{t}+\operatorname{div} \mathbf{p}=f, & (\mathbf{x}, t) \in \Omega \times(0, T], \\
\text { (c) } u(\mathbf{x}, t)=0, & (\mathbf{x}, t) \in \partial \Omega \times(0, T], \\
\text { (d) } u(\mathbf{x}, 0)=u_{0}(\mathbf{x}), & \mathbf{x} \in \Omega .
\end{array}
$$

The pseudo-parabolic integro-differential equation is an important integro-differential equation because of its wide application in many practical problems such as fluid mechanics, nuclear dynamics, or biomechanics. The existence and uniqueness problem of the solution has been done in $[6,14,10,11]$.

Recently, some numerical methods of (1.1) or (1.2) are studied by several authors([16,3]). In [16], Zhou et al. studied a $H^{1}$-Galerkin mixed finite element method of the problem (1.1) and proved the optimal convergence of the method. In [3], Che studied the mixed finite element method of (1.2) and obtained the optimal error estimates of this mixed finite element scheme in the $\mathbf{H}(\operatorname{div} ; \Omega)$-norm and $L^{2}$-norm.

The purpose of this paper is to study the mixed covolume method for the problems (1.2). Mixed 
covolume method was first proposed by Russell([13]). The basic technique of this method was to relate the Petro-Galerkin scheme to a standard finite element Galerkin or mixed method through an introduction of the transfer operator $\gamma h$ that maps the trial function space into the test function space. This method not only preserves the simplicity of finite difference and the high accuracy of finite element but maintains the mass conservation law, which is very important to fluid and under-ground fluid computations. The optimal convergence of the mixed covolume method for linear elliptic problems on triangular grids was given by Chou et al.([4]), and Yang et al.([15]) extended this numerical method to the parabolic problem.

In a mixed covolume method for differential systems (1.2) one uses two staggered irregular gridsa primal grid consisting of primal volumes (elements) and a dual grid consisting of covolumes (dual elements). The associated discretization equations are derived by integrating the differential equations over the volumes and using the divergence theorem or the Stokes theorem when proper. The balance between the numbers of unknowns and equations depends on a judicious placement of the degrees of freedom for the unknown functions.

The goal of this article is to consider the error estimates of this mixed covolume scheme. We give the approximate solution with optimal accuracy in $\mathbf{H}(\operatorname{div} ; \Omega) \times L^{2}(\Omega)$. Hence we give the following assumptions.

Assumption 1. We suppose that

$$
\begin{array}{lc}
\text { (a) } 0<\frac{1}{c_{2}} \leq \alpha, & \alpha \in L^{\infty}\left(0, T ; W^{1, \infty}(\Omega)\right), \\
\text { (b) } \beta, \gamma \in L^{\infty}\left(0, T ;\left(W^{1, \infty}(\Omega)\right)^{2}\right), & b, c \in L^{\infty}\left(0, T ; W^{1, \infty}(\Omega)\right), \\
\text { (c) } u_{0} \in H^{1}(\Omega), & f \in L^{\infty}\left(0, T ; L^{2}(\Omega)\right) .
\end{array}
$$

The organization of this paper is as follows. In the next section we describe the mixed covolume method for the problem (1.1) on triangles. In section 3, we introduce a generalized mixed covolume elliptic projection associated with (1.2) and study the error estimates of the generalized mixed covolume elliptic projection. In section 4, using the error estimates obtained in section 3 , we establish the optimal rate of convergence for the approximate solution in the $\mathbf{H}(\operatorname{div} ; \Omega)$-norm and $L^{2}$-norm.

Throughout this paper, we use $C$ (without or with subscript) to denote a generic constant independent of the discretization parameters, which has different values in different appearances. We also adopt the standard definitions and notations of Sobolev spaces and their full norms and seminorms in [1], [5], [9].

\section{Mixed covolume formulation}

For the problem (1.2), we adopt $\mathbf{V} \times W$ as the weak solution space, where the space $W=\left\{u \in L^{2}(\Omega): u=0\right.$ on $\left.\partial \Omega\right\}$ and the space $\mathbf{V}=\mathbf{H}(\operatorname{div} ; \Omega)$ is the set of all vector-valued functions $\mathbf{v} \in L^{2}(\Omega)^{2}$ such that $\operatorname{div} \mathbf{v} \operatorname{div} \mathbf{v} \in L^{2}(\Omega)$ and its norm is defined as

$$
\|\mathbf{v}\|_{H(\text { div) }}^{2}=\|\mathbf{v}\|^{2}+\|\operatorname{div} \mathbf{v}\|^{2} \text {. }
$$

Noting that $\left.u(\mathbf{x}, t)\right|_{\partial \Omega}=0$ and $\left.u_{t}(\mathbf{x}, t)\right|_{\partial \Omega}=0$, the weak formulation associated with (1.2) is to find $(\mathbf{p}, u):[0, T] \rightarrow \mathbf{V} \times W$ such that

$$
\begin{array}{ll}
\text { (a) }(a \mathbf{p}, \mathbf{v})+\left(u_{t}+b u+\int_{0}^{t} c u d \tau, \operatorname{div} \mathbf{v}\right)+\left(\beta u+\int_{0}^{t} \gamma u d \tau, \mathbf{v}\right)=0, & \forall \mathbf{v} \in \mathbf{V}, 0<t \leq T, \\
\text { (b) }\left(u_{t}, w\right)+(\operatorname{div} \mathbf{p}, w)=(f, w), & \forall w \in W, 0<t \leq T, \\
\text { (c) }(u(0), w)=\left(u_{0}, w\right) & \forall w \in W,
\end{array}
$$

where $(\cdot, \cdot)$ is the $\left(L^{2}\right)^{2}$ or $L^{2}$-inner product.

In order to describe the mixed covolume method for the problem (1.2), we first construct the partition $T_{h}$ of the domain $\Omega$ and the trial function space. Referring to Fig.1, let $T_{h}=\bigcup K_{B}$ be a quasi-uniform (regular) triangulation of the domain $\Omega$, where $K_{B}$ is the triangle with the barycenter $B$, 
and $h=\max h_{K_{B}}, h_{K_{B}}$ is the diameter of the triangle $K_{B}$. The nodes of a triangular element $K_{B}$ are the midpoints of the edges of $K_{B}$. Let $P_{1}, P_{2}, \cdots, P_{N_{s}}$ denote the nodes belonging to the interior of $\Omega$ and $P_{N_{s+1}}, \cdots, P_{N}$ the nodes on the boundary $\partial \Omega$. Based on the partition $T_{h}$, we select the lowest-order Raviart-Thomas mixed space $\mathbf{V}_{h} \times W_{h}$ as the trial function space, where the spaces

$$
\mathbf{V}_{h}=\left\{\mathbf{v}_{h} \in \mathbf{H}(\operatorname{div} ; \Omega):\left.\mathbf{v}_{h}\right|_{K}=(a+b x, c+b y), \forall K \in T_{h}\right\}
$$

and

$$
W_{h}=\left\{w_{h} \in W:\left.w_{h}\right|_{k}=\text { const, } \forall K \in T_{h}\right\} .
$$

For any $\mathbf{w}_{h}=\left(w_{1 h}, w_{2 h}\right) \in \mathbf{V}_{h}$, we define the discrete seminorm and norm as

$$
\left|\mathbf{w}_{h}\right|_{1, h}^{2}=\sum_{K \in T_{h}}\left(\left|\nabla w_{1 h}\right|_{0, K}^{2}+\left|\nabla w_{2 h}\right|_{0, K}^{2}\right), \quad\left\|\mathbf{w}_{h}\right\|_{1, h}^{2}=\left\|\mathbf{w}_{h}\right\|^{2}+\left|\mathbf{w}_{h}\right|_{1, h}^{2} .
$$

We introduce the Raviart-Thomas projection $\prod_{h}^{[12,8]}: \mathbf{V} \rightarrow \mathbf{V}_{h}$ satisfying

$$
\left(\operatorname{div}\left(\mathbf{p}-\Pi_{h} \mathbf{p}\right), w_{h}\right)=0, \forall w_{h} \in W_{h},
$$

and the $L^{2}$ orthogonal projection $P_{h}: W \rightarrow W_{h}$ satisfying

$$
\left(w_{h}, P_{h} u-u\right)=0 \quad \forall w_{h} \in W_{h} .
$$

Then the following properties of the projections $\Pi_{h}$ and $P_{h}$ hold ${ }^{[12]}$ :

$$
\begin{aligned}
& \left\|\mathbf{p}-\prod_{h} \mathbf{p}\right\| \leq C h\|\mathbf{p}\|_{1}, \quad \forall \mathbf{p} \in\left(H^{1}(\Omega)\right)^{2}, \\
& \left\|\operatorname{div}\left(\mathbf{p}-\prod_{h} \mathbf{p}\right)\right\| \leq C h^{l}\|\operatorname{div} \mathbf{p}\|_{l}, l=0,1 \quad \forall \operatorname{div} \mathbf{p} \in H^{1}(\Omega) \text {, } \\
& \left\|P_{h} u\right\| \leq C\|u\|, \quad \forall u \in W, \\
& \left\|P_{h} u-u\right\|_{-1}+h\left\|P_{h} u-u\right\| \leq C h^{2}\|u\|_{1}, \quad \forall u \in H^{1}(\Omega), \\
& \left\|P_{h} u-u\right\|_{0, \infty} \leq C h\|u\|_{1, \infty}, \forall u \in W^{1, \infty}(\Omega) .
\end{aligned}
$$

Next, we construct the dual partition $T_{h}^{*}=\bigcup K_{p}^{*}$ and the test function space. Choose the barycenter $B$ of $K_{B} \in T_{h}$ and connect it with three vertices of the element $K_{B}$. Then wepartition $K_{B}$ into three subtriangles. For any interior node $P$, which is the midpoint of the edge $e$, the dual element $K_{P}^{*}$ is the quadrilateral consisting of the two subtriangles which have $e$ as their common edge. For any node $P$ on the boundary $\partial \Omega$, the corresponding dual element is the subtriangle, where $P$ is one of the midpoints of its edges. The dual partition $T_{h}^{*}$ is the union of the interior quadrilaterals and the border triangles. Referring to Fig. 1 , the interior node $P_{1}$ belongs to the common side of the triangles $K_{B_{1}}=\Delta A_{1} A_{2} A_{3}$ and $K_{B_{2}}=\Delta A_{1} A_{3} A_{5}$ and the quadrilateral $A_{1} B_{1} A_{3} B_{2}$ is the dual element $K_{P_{1}}^{*}$ with node $P_{1}$. For a boundary node like $P_{6}$ the associated dual element $K_{P_{6}}^{*}$ is a triangle ( $\triangle A_{5} B_{3} A_{4}$ in this case). In general, let $K_{P}^{*}=K_{P L} \cup K_{P R}$, where when $P$ is a interior node, for example $P_{1}, K_{P_{1} L}=\triangle A_{1} B_{1} A_{3}$ and $K_{P_{1} R}=\triangle A_{1} A_{3} B_{2}$, and $K_{P}^{*}=K_{P L}$ when $P$ is a boundary node. Define the transfer operator $\gamma_{h}^{[4]}: \mathbf{V}_{h} \rightarrow L^{2}(\Omega)^{2}:$

$$
\gamma_{h} \mathbf{v}_{h}=\sum_{j=1}^{N}\left(\left.\mathbf{v}_{h}\right|_{K_{P_{j} L}}\left(P_{j}\right) \chi_{K_{P_{j} L}}^{*}+\left.\mathbf{v}_{h}\right|_{K_{P_{j} R}}\left(P_{j}\right) \chi_{K_{P_{j} R}}^{*}\right), \forall \mathbf{v} \in \mathbf{V}_{h},
$$

where $\chi_{Q}^{*}$ is the characteristic function of the set $Q$. Now let us define the test function space associated with the dual partition to be $\mathbf{Y}_{h} \times L_{h}$, where $\mathbf{Y}_{h}=R\left(\gamma_{h}\right)=$ the range of $\gamma_{h}$. 


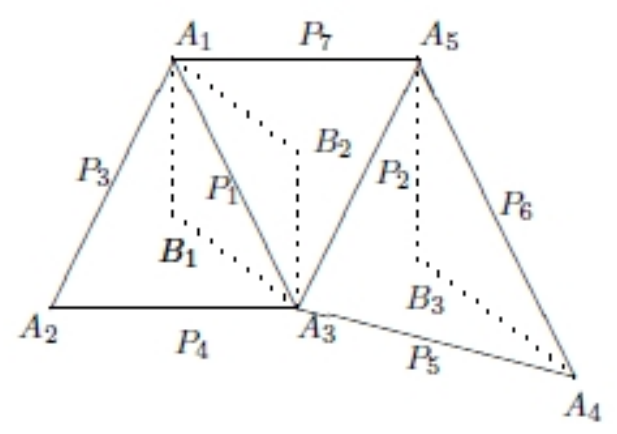

Fig.1 Primal and Dual Domains

From the definition of the operator $\gamma_{h}$, we know that the function $\mathbf{w}_{h} \in \mathbf{Y}_{h}$ is a piecewise constant vector function. It takes on different constant vectors on the left and right parts of an interior dual element. Note that the two constant vectors $\left.\mathbf{w}_{h}\right|_{K_{P L}}$ and $\left.\mathbf{w}_{h}\right|_{K_{P R}}$ must satisfy $\left.\mathbf{w}_{h}\right|_{K_{P L}} \cdot \mathbf{n}=\left.\mathbf{w}_{h}\right|_{K_{P R}} \cdot \mathbf{n}$ where $\mathbf{n}$ is a fixed normal unit vector to the common edge of $K_{P L}$ and $K_{P R}$. Obviously, the transfer operator $\gamma_{h}$ sets up a one-to-one correspondence between thetrial and the test spaces and $\operatorname{dim} \mathbf{Y}_{h}=\operatorname{dim} \mathbf{V}_{h}$.

Finally, we construct the mixed covolume method for the problem (1.2). For $t \in(0, T]$,integrating the equation $(a)$ in (1.2) on the dual domain $K_{P}^{*}$ and applying Green's formula, we get that

$$
\begin{aligned}
0 & =\int_{K_{P}^{*}}\left(\alpha \mathbf{p}+\nabla u_{t}+\nabla(b u)+\beta u+\int_{0}^{t} \nabla(c u) \mathrm{d} \tau+\int_{0}^{t} \gamma u \mathrm{~d} \tau\right) \mathrm{dx} \\
& =\int_{K_{P}^{*}}\left(\alpha \mathbf{p}+\beta u+\int_{0}^{t} \gamma u \mathrm{~d} \tau\right) \mathrm{dx}+\int_{\partial K_{P}^{*}}\left(u_{t}+b u+\int_{0}^{t} c u \mathrm{~d} \tau\right) \mathbf{n} \mathrm{d} s,
\end{aligned}
$$

where $\mathbf{n}$ stands for the unit outer normal direction of $\partial K_{P}^{*}$. Noting that the boundary condition $\left.u(\mathbf{x}, t)\right|_{\partial \Omega}=0$ implies $\left.u_{t}(\mathbf{x}, t)\right|_{\partial \Omega}=0,(2.11)$ can be rewritten as

$$
\int_{K_{P}^{*}}\left(\alpha \mathbf{p}+\beta u+\int_{0}^{t} \gamma u \mathrm{~d} \tau\right) \mathrm{d} \mathrm{x}+\int_{\partial K_{P}^{*} \backslash \partial \Omega}\left(u_{t}+b u+\int_{0}^{t} c u \mathrm{~d} \tau\right) \mathbf{n} \mathrm{d} s=0 .
$$

Integrating the equation $(b)$ in (1.2) on the primal element $K=K_{B}$, we obtain that

$$
\int_{K}\left(u_{t}+\operatorname{div} \mathbf{p}\right) \mathbf{d} \mathbf{x}=\int_{K} f \mathrm{~d} \mathbf{x} .
$$

For $\mathbf{v}=\left(v_{1}, v_{2}\right) \in \mathbf{Y}_{h}, u \in L^{2}(\Omega)$, let

$$
B(\mathbf{v}, u)=\sum_{j=1}^{N_{s}}\left\{\int_{\partial K_{P_{j}}^{*} \cap K_{P_{j} L}} u \mathbf{n}_{j} \cdot \mathbf{v} \mathrm{ds}+\int_{\partial K_{P_{j}}^{*} \cap K_{P_{j} R} R} u \mathbf{n}_{j} \cdot \mathbf{v} \mathrm{ds}\right\},
$$

where $\mathbf{n}_{j}$ stands for the unite outer normal direction of $K_{P_{j}}^{*}, j=1, \cdots, N_{s}$. Using the bilinear form $B(\cdot ; \cdot)$ and the transfer operator $\gamma_{h},(2.12)$ and (2.13) can be rewritten as

$$
\begin{array}{ll}
\text { (a) }\left(\alpha \mathbf{p}+\beta u+\int_{0}^{t} \gamma u d \tau, \gamma_{h} \mathbf{v}_{h}\right)+B\left(\gamma_{h} \mathbf{v}_{h}, u_{t}+b u+\int_{0}^{t} c u d \tau\right)=0, & \forall \mathbf{v}_{h} \in \mathbf{V}_{h}, \\
\text { (b) }\left(u_{t}, w_{h}\right)+\left(\operatorname{div} \mathbf{p}, w_{h}\right)=\left(f, w_{h}\right), & \forall w_{h} \in \mathbf{W}_{h},
\end{array}
$$

Replacing $\mathbf{p}$ and $u$ in (2.15) by their approximations $\mathbf{p}_{h}$ and $u_{h}$, we construct the semidiscrete mixed covolume scheme for the problem (1.1) (or (1.2)) as: Find $\left\{\mathbf{p}_{h}, u_{h}\right\}:[0, T] \rightarrow \mathbf{V}_{h} \times W_{h}$ such that

(a) $\left(\alpha \mathbf{p}_{h}+\beta u_{h}+\int_{0}^{t} \gamma u_{h} d \tau, \gamma_{h} \mathbf{v}_{h}\right)+B\left(\gamma_{h} \mathbf{v}_{h}, u_{h t}+b u_{h}+\int_{0}^{t} c u_{h} d \tau\right)=0, \forall \mathbf{v}_{h} \in \mathbf{V}_{h}, t \in[0, T]$,

(b) $\left(u_{h t}, w_{h}\right)+\left(\operatorname{div} \mathbf{p}_{h}, w_{h}\right)=\left(f, w_{h}\right)$,

(c) $\left(u_{h}(0), w_{h}\right)=\left(u_{0}, w_{h}\right)$,

$$
\begin{aligned}
& \forall w_{h} \in \mathbf{W}_{h}, t \in[0, T], \\
& \forall w_{h} \in \mathbf{W}_{h} .
\end{aligned}
$$

From [4] and [15], the operator $\gamma_{h}$ has the following properties. 
Lemma 2.1 ${ }^{[4]}$ For any function $\mathbf{v}_{h} \in \mathbf{V}_{h}$ and $w_{h} \in W_{h}$, the following relations

$$
\begin{gathered}
B\left(\gamma_{h} \mathbf{v}_{h}, w_{h}\right)=-\left(\operatorname{div} \mathbf{v}_{h}, w_{h}\right), \\
\left\|\gamma_{h} \mathbf{v}_{h}\right\|=\left\|\mathbf{v}_{h}\right\|
\end{gathered}
$$

hold.

Lemma 2.2 ${ }^{[15]}$ There exists a positive constant $C_{1}$ independent of $h$ such that

$$
\left\|\alpha \mathbf{v}_{h}, \gamma_{h} \mathbf{v}_{h}\right\| \geq \frac{1}{C_{1}}\left\|\mathbf{v}_{h}\right\|, \forall \mathbf{v}_{h} \in \mathbf{V}_{h} .
$$

Lemma 2.3 ${ }^{[15]}$ For any functions $\mathbf{v}_{h}, \mathbf{q}_{h} \in \mathbf{V}_{h}$, the following symmetry relation

$$
\left\|\gamma_{h} \mathbf{q}_{h}, \mathbf{v}_{h}\right\|=\left\|\mathbf{q}_{h}, \gamma_{h} \mathbf{v}_{h}\right\|
$$

holds.

Lemma 2.4 ${ }^{[15]}$ There exists a positive constant $C$ independent of $h$ such that

$$
\begin{gathered}
\left\|\left(I-\gamma_{h}\right) \mathbf{v}_{h}\right\| \leq C h\left\|\mathbf{v}_{h}\right\|_{1, h}, \forall \mathbf{v}_{h} \in \mathbf{V}_{h} \\
\left|\left(\alpha \mathbf{q}_{h},\left(I-\gamma_{h}\right) \mathbf{v}_{h}\right)\right| \leq C h\left\|\mathbf{q}_{h}\right\|_{1, h}\left\|\mathbf{v}_{h}\right\|, \forall \mathbf{q}_{h}, \mathbf{v}_{h} \in \mathbf{V}_{h} \\
\left|\left(\alpha \mathbf{q},\left(I-\gamma_{h}\right) \mathbf{v}_{h}\right)\right| \leq C h\|\mathbf{q}\|_{1}\left\|\mathbf{v}_{h}\right\|, \forall \mathbf{q} \in\left(H^{1}(\Omega)\right)^{2}, \mathbf{v}_{h} \in \mathbf{V}_{h}
\end{gathered}
$$

Lemma 2.5 ${ }^{[15]}$ There exists a positive constant $C$ independent of $h$ such that

$$
\left\|\mathbf{q}-\gamma_{h} \Pi_{h} \mathbf{q}\right\|_{0, q} \leq C h\|\mathbf{q}\|_{1, q}, \forall \mathbf{q} \in\left(W^{1, q}\right)^{2}, 1<q<\infty .
$$

Theorem 2.6 There exists a unique solution $\left\{\mathbf{p}_{h}, u_{h}\right\}$ in $\mathbf{V}_{h} \times W_{h}$ for the system (2.16).

Proof. Since the system (2.16) is linear, it suffices to show that the associated homogeneous system

(a) $\left(\alpha \mathbf{p}_{h}+\beta u_{h}+\int_{0}^{t} \gamma u_{h} d \tau, \gamma_{h} \mathbf{v}_{h}\right)+B\left(\gamma_{h} \mathbf{v}_{h}, u_{h t}+b u_{h}+\int_{0}^{t} c u_{h} d \tau\right)=0, \forall \mathbf{v}_{h} \in \mathbf{V}_{h}, t \in[0, T]$,

(b) $\left(u_{h t}, w_{h}\right)+\left(\operatorname{div} \mathbf{p}_{h}, w_{h}\right)=\left(f, w_{h}\right), \quad \forall w_{h} \in \mathbf{W}_{h}, t \in[0, T]$,

(c) $\left(u_{h}(0), w_{h}\right)=0$,

$$
\forall w_{h} \in \mathbf{W}_{h} \text {. }
$$

has only the trivial solution.

In fact, choosing $\mathbf{v}_{h}=\mathbf{p}_{h}$ in (a) equation and in (b) equation in (2.25) and using (2.17), we have that

$$
\begin{aligned}
\left(\alpha \mathbf{p}_{h}, \gamma_{h} \mathbf{p}_{h}\right)+\left(u_{h t}, u_{h t}\right)= & -\left(\beta u_{h}+\int_{0}^{t} \gamma u_{h} d \tau, \gamma_{h} \mathbf{p}_{h}\right)-\left(u_{h t},\left(P_{h} b\right) u_{h}+\int_{0}^{t}\left(P_{h} c\right) u_{h} d \tau\right) \\
& -B\left(\gamma_{h} \mathbf{p}_{h},\left(b-P_{h} b\right) u_{h}+\int_{0}^{t}\left(c-P_{h} c\right) u_{h} d \tau\right)=A
\end{aligned}
$$

Lemma 2.2 implies that

$$
\left(\alpha \mathbf{p}_{h}, \gamma_{h} \mathbf{p}_{h}\right)+\left(u_{h t}, u_{h t}\right) \geq \frac{1}{C_{1}}\left\|\mathbf{p}_{h}\right\|^{2}+\left\|u_{h t}\right\|^{2}
$$

Noticing (2.18) and the Lemma 4.2 in the four section of this paper, we obtain that

$$
\begin{aligned}
|A| & \leq C(1+h)\left(\left\|u_{h}\right\|+\int_{0}^{t}\left\|u_{h}\right\| d \tau\right)\left(\left\|\mathbf{p}_{h}\right\|+\left\|u_{h t}\right\|\right) \\
& \leq C\left(\left\|u_{h}\right\|+\int_{0}^{t}\left\|u_{h}\right\| d \tau\right)^{2}+\frac{1}{2 C_{1}}\left\|\mathbf{p}_{h}\right\|^{2}+\frac{1}{2}\left\|u_{h t}\right\|^{2}
\end{aligned}
$$

Combining (2.26) with (2.27) and (2.28), we have that

$$
\left\|\mathbf{p}_{h}\right\|+\left\|u_{h t}\right\| \leq C\left(\left\|u_{h}\right\|+\int_{0}^{t}\left\|u_{h}\right\| d \tau\right)
$$

(c) equation in (2.25) and (2.29) implies that

$$
\left\|u_{h}\right\| \leq C \int_{0}^{t}\left\|u_{h t}\right\| d \tau \leq C \int_{0}^{t}\left\|u_{h}\right\| d \tau
$$

Using Gronwall's inequality we have $\left\|u_{h}\right\|=0$, which and (2.29) implies that $\left\|\mathbf{p}_{h}\right\|=0$. Hence we have $u_{h}=0$ and $\mathbf{p}_{h}=\mathbf{0}$. This completes the proof of the lemma.

By Lemma 2.1, we know that 


$$
\begin{aligned}
B\left(\gamma_{h} \mathbf{v}_{h}, u_{h t}+b u_{h}+\int_{0}^{t} c u_{h} d \tau\right)= & -\left(\operatorname{div} \mathbf{v}_{h}, u_{h t}+\left(P_{h} b\right) u_{h}+\int_{0}^{t}\left(P_{h} c\right) u_{h} d \tau\right) \\
& +B\left(\gamma_{h} \mathbf{v}_{h},\left(b-P_{h} b\right) u_{h}+\int_{0}^{t}\left(c-P_{h} c\right) u_{h} d \tau\right)
\end{aligned}
$$

which implies that the mixed covolume method (2.16) can be rewritten as: Find $\left\{\mathbf{p}_{h}, u_{h}\right\}:[0, T] \rightarrow \mathbf{V}_{h} \times W_{h}$ such that

(a) $\left(\alpha \mathbf{p}_{h}+\beta u_{h}+\int_{0}^{t} \gamma u_{h} d \tau, \gamma_{h} \mathbf{v}_{h}\right)-\left(\operatorname{div} \mathbf{v}_{h}, u_{h t}+\left(P_{h} b\right) u_{h}+\int_{0}^{t}\left(P_{h} c\right) u_{h} d \tau\right)$

$$
+B\left(\gamma_{h} \mathbf{v}_{h},\left(b-P_{h} b\right) u_{h}+\int_{0}^{t}\left(c-P_{h} c\right) u_{h} d \tau\right)=0, \forall \mathbf{v}_{h} \in \mathbf{V}_{h}, t \in[0, T],
$$

(b) $\left(u_{h t}, w_{h}\right)+\left(\operatorname{div} \mathbf{p}_{h}, w_{h}\right)=\left(f, w_{h}\right)$,

$\forall w_{h} \in \mathbf{W}_{h}, t \in[0, T]$,

(c) $\left(u_{h}(0), w_{h}\right)=\left(u_{0}, w_{h}\right)$,

$$
\forall w_{h} \in \mathbf{W}_{h} \text {. }
$$

\section{Generalized Mixed Covolume Elliptic Projection}

In the study of mixed covolume methods for parabolic problems, we usually introduce a mixed covolume elliptic projection associated with our equations. Modifying this idea according to our pseudo-parabolic integro-differential equations, we define a map $\left\{\mathbf{p}_{h}, u_{h}\right\}:[0, T] \rightarrow \mathbf{V}_{h} \times W_{h}$ such that

(a) $\left(\alpha\left(\tilde{\mathbf{p}}_{h}-\mathbf{p}\right), \gamma_{h} \mathbf{v}_{h}\right)-\left(\operatorname{div} \mathbf{v}_{h},\left(\tilde{u}_{h t}-u_{t}\right)+b\left(\tilde{u}_{h}-u\right)+\int_{0}^{t} c\left(\tilde{u}_{h}-u\right) d \tau\right)$

$$
\begin{array}{cc}
+\left(\beta\left(\tilde{u}_{h}-u\right)+\int_{0}^{t} \gamma\left(\tilde{u}_{h}-u\right) d \tau, \gamma_{h} \mathbf{v}_{h}\right)=\left(\alpha \mathbf{p},\left(I-\gamma_{h}\right) \mathbf{v}_{h}\right)+\left(\beta u+\int_{0}^{t} \gamma u d \tau,\left(I-\gamma_{h}\right) \mathbf{v}_{h}\right), \\
& \forall \mathbf{v}_{h} \in \mathbf{V}_{h}, t \in(0, T], \\
\text { (b) }\left(\operatorname{div}\left(\tilde{\mathbf{p}}_{h}-\mathbf{p}\right), w_{h}\right)=0, & \forall w_{h} \in \mathbf{W}_{h}, t \in(0, T], \\
\text { (c) }\left(\tilde{u}_{h}(0), w_{h}\right)=\left(u_{0}, w_{h}\right), & \forall w_{h} \in \mathbf{W}_{h} .
\end{array}
$$

Before collecting the results of the error analysis of $\tilde{u}_{h}$ and $\tilde{\mathbf{p}}_{h}$, let us demonstrate the existence and uniqueness of the solution of (3.1). In fact, it suffices to show that the associated homogeneous system

(a) $\left(\alpha \tilde{\mathbf{p}}_{h}, \gamma_{h} \mathbf{v}_{h}\right)-\left(\operatorname{div} \mathbf{v}_{h}, \tilde{u}_{h t}+b \tilde{u}_{h}+\int_{0}^{t} c \tilde{u}_{h} d \tau\right)+\left(\beta \tilde{u}_{h}+\int_{0}^{t} \gamma \tilde{u}_{h} d \tau, \gamma_{h} \mathbf{v}_{h}\right)=0, \quad \forall \mathbf{v}_{h} \in \mathbf{V}_{h}, t \in[0, T]$,

(b) $\left(\operatorname{div} \tilde{\mathbf{p}}_{h}, w_{h}\right)=0$,

$\forall w_{h} \in \mathbf{W}_{h}, t \in[0, T]$,

(c) $\left(\tilde{u}_{h}(0), w_{h}\right)=0$,

has only the trivial solution. Taking $w_{h}=\operatorname{div} \tilde{\mathbf{p}}_{h}$ in $(b)$ equation and $\tilde{\mathbf{v}}_{h}=\mathbf{p}_{h}$ in $(a)$ equation in (3.2), we have by using Lemma 2.2 and Lemma 2.1 that

$$
\frac{1}{C_{1}}\left\|\tilde{\mathbf{p}}_{h}\right\|^{2} \leq\left(\alpha \tilde{\mathbf{p}}_{h}, \gamma_{h} \tilde{\mathbf{p}}_{h}\right)=-\left(\beta \tilde{u}_{h}+\int_{0}^{t} \gamma \tilde{u}_{h} d \tau, \gamma_{h} \tilde{\mathbf{p}}_{h}\right) \leq C\left(\left\|\tilde{u}_{h}\right\|+\int_{0}^{t}\left\|\tilde{u}_{h}\right\| d \tau\right)\left\|\mathbf{p}_{h}\right\|,
$$

which implies that

$$
\left\|\tilde{\mathbf{p}}_{h}\right\|^{2} \leq C\left(\left\|\tilde{u}_{h}\right\|+\int_{0}^{t}\left\|\tilde{u}_{h}\right\| d \tau\right) .
$$

On the other hand, from [11] we know that for $\mathbf{V}_{h} \times W_{h}$ there exists a positive constant $C$ independent of $h$ such that

$$
\left\|w_{h}\right\| \leq C \sup _{\mathbf{v}_{h} \in \mathbf{V}_{h}, \mathbf{v}_{h} \neq \mathbf{0}} \frac{\left(w_{h}, \operatorname{div} \mathbf{v}_{h}\right)}{\left\|\mathbf{v}_{h}\right\|_{\mathbf{H}(\operatorname{div})}}
$$

Hence we have from (3.4) and (a) equation in (3.2), (2.18), (2.1), (3.3) that 


$$
\begin{aligned}
\left\|\tilde{u}_{h t}\right\| & \leq \sup _{\mathbf{v}_{h} \in \mathbf{V}_{h}, \mathbf{v}_{h} \neq \mathbf{0}} \frac{\left(\tilde{u}_{h t}, \operatorname{div} \mathbf{v}_{h}\right)}{\left\|\mathbf{v}_{h}\right\|_{\mathbf{H}(\text { div) }}} \\
& \leq C \sup _{\mathbf{v}_{h} \in \mathbf{V}_{h}, \mathbf{v}_{h} \neq \mathbf{0}} \frac{\left(\alpha \tilde{\mathbf{p}}_{h}+\beta \tilde{u}_{h}+\int_{0}^{t} \gamma \tilde{u}_{h} d \tau, \gamma_{h} \mathbf{v}_{h}\right)-\left(b \tilde{u}_{h}+\int_{0}^{t} c \tilde{u}_{h} d \tau, \operatorname{div} \mathbf{v}_{h}\right)}{\left\|\mathbf{v}_{h}\right\|_{\mathbf{H}(\text { div) }}} \\
& \leq C \sup _{\mathbf{v}_{h} \in \mathbf{V}_{h}, \mathbf{v}_{h} \neq \mathbf{0}} \frac{\left(\left\|\tilde{\mathbf{p}}_{h}\right\|+\left\|\tilde{u}_{h}\right\|+\int_{0}^{t}\left\|\tilde{u}_{h}\right\| d \tau\right)\left\|\mathbf{v}_{h}\right\|_{\mathbf{H}(\text { div })}}{\left\|\mathbf{v}_{h}\right\|_{\mathbf{H}(\text { div) }}} \\
& \leq C\left(\left\|\tilde{u}_{h}\right\|+\int_{0}^{t}\left\|\tilde{u}_{h}\right\| d \tau\right)
\end{aligned}
$$

Similarly to the proof of the Theorem 2.6, we have $\tilde{u}_{h}=0$ and $\tilde{\mathbf{p}}_{h}=\mathbf{0}$. Hence the existence and uniqueness of the solution of (3.1) has been demonstrated and $\left(\tilde{\mathbf{p}}_{h}, \tilde{u}_{h}\right)$ in (3.1) is well defined.

Now, let us study some properties of $\left\{\tilde{\mathbf{p}}_{h}, \tilde{u}_{h}\right\}$. Let $\tilde{\xi}=\tilde{\mathbf{p}}_{h}-\mathbf{p}, \tilde{\tau}=\tilde{u}_{h}-P_{h} u$.Noting that (2.5), then (3.1) can be rewritten as

(a) $\left(\alpha \tilde{\xi}, \gamma_{h} \mathbf{v}_{h}\right)-\left(\operatorname{div} \mathbf{v}_{h}, \tilde{\tau}_{t}+b \tilde{\tau}+\int_{0}^{t} c \tilde{\tau} d \tau\right)+\left(\beta \tilde{\tau}+\int_{0}^{t} \gamma \tilde{\tau} d \tau, \gamma_{h} \mathbf{v}_{h}\right)$

$$
\begin{array}{ll}
=\left(\alpha \mathbf{p},\left(I-\gamma_{h}\right) \mathbf{v}_{h}\right)+\left(\beta u+\int_{0}^{t} \gamma u d \tau,\left(I-\gamma_{h}\right) \mathbf{v}_{h}\right)+\left(b\left(P_{h} u-u\right)+\int_{0}^{t} c\left(P_{h} u-u\right) d \tau, \operatorname{div} \mathbf{v}_{h}\right) \\
-\left(\beta\left(P_{h} u-u\right)+\int_{0}^{t} \gamma\left(P_{h} u-u\right) d \tau, \gamma_{h} \mathbf{v}_{h}\right), & \forall \mathbf{v}_{h} \in \mathbf{V}_{h}, t \in(0, T], \\
\text { (b) }\left(\operatorname{div} \xi, w_{h}\right)=0, & \forall w_{h} \in \mathbf{W}_{h}, t \in(0, T], \\
\text { (c) }\left(\tilde{\tau}(0), w_{h}\right)=0, & \forall w_{h} \in \mathbf{W}_{h} .
\end{array}
$$

Lemma 3.1. Suppose that $\beta, \gamma \in L^{\infty}\left(0, T ;\left(L^{\infty}(\Omega)\right)^{2}\right)$ and $u \in L^{\infty}\left(0, T ; H^{1}(\Omega)\right)$. Then for any $\varsigma \in\left(H^{1}(\Omega)\right)^{2}$ and $t \in(0, T]$, we have

$$
\begin{gathered}
\left|\left(\beta\left(P_{h} u-u\right), \gamma_{h} \Pi_{h} \varsigma\right)\right| \leq C h^{2}\|u\|_{1}\|\varsigma\|_{1}, \\
\left|\left(\int_{0}^{t} \gamma\left(P_{h} u-u\right) d \tau, \gamma_{h} \Pi_{h} \varsigma\right)\right| \leq C h^{2} \int_{0}^{t}\|u\|_{1} d \tau\|\varsigma\|_{1} .
\end{gathered}
$$

Proof. The proof of (3.7) see lemma 4.1 in [15]. To prove (3.8), it follows (2.9) and (2.24) that

$$
\begin{aligned}
& \left|\left(\int_{0}^{t} \gamma\left(P_{h} u-u\right) d \tau, \gamma_{h} \Pi_{h} \varsigma\right)\right|=\left|\left(\int_{0}^{t} \gamma\left(P_{h} u-u\right) d \tau, \gamma_{h} \Pi_{h} \varsigma-\varsigma\right)\right|+\left|\left(\int_{0}^{t} \gamma\left(P_{h} u-u\right) d \tau, \varsigma\right)\right| \\
& \quad \leq C \int_{0}^{t}\left\|P_{h} u-u\right\| d \tau\left\|\gamma_{h} \Pi_{h} \varsigma-\varsigma\right\|+C \int_{0}^{t}\left\|P_{h} u-u\right\|_{-1} d \tau\|\varsigma\|_{1} \leq C h^{2} \int_{0}^{t}\|u\|_{1} d \tau\|\varsigma\|_{1}
\end{aligned}
$$

This completes the proof of (3.8).

Lemma 3.2. Suppose that $\alpha \in L^{\infty}\left(0, T ; W^{1, \infty}(\Omega)\right)$ and $\beta, \gamma \in L^{\infty}\left(0, T ;\left(W^{1, \infty}(\Omega)\right)^{2}\right)$, $\mathbf{p} \in L^{\infty}\left(0, T ;\left(H^{1}(\Omega)\right)^{2}\right), u \in L^{\infty}\left(0, T ; H^{1}(\Omega)\right)$. Then for any $\zeta \in\left(H^{1}(\Omega)\right)^{2}$ and $t \in(0, T]$, we have

$$
\left|\left(\alpha \mathbf{p}+\beta u+\int_{0}^{t} \gamma u d \tau,\left(I-\gamma_{h}\right) \prod_{h} \zeta\right)\right| \leq C h^{2}\left(\|\mathbf{p}\|_{1}+\|u\|_{1}+\int_{0}^{t}\|u\|_{1} d \tau\right)\|\zeta\|_{1}
$$

Proof. Letting $\mathbf{v}=\alpha \mathrm{p}+\beta u+\int_{0}^{t} \gamma u d \tau$, and $\overline{\mathbf{v}}$ be a piecewise constant approximation to $\mathbf{v}$, from (2.6) and (2.24) we obtain that

$$
\begin{aligned}
\mid(\alpha \mathbf{p}+\beta u+ & \left.\int_{0}^{t} \gamma u d \tau,\left(I-\gamma_{h}\right) \prod_{h} \zeta\right)|=|\left(\mathbf{v}, \prod_{h} \zeta-\gamma_{h} \prod_{h} \zeta\right) \mid \\
& =\left|\left(\mathbf{v}-\overline{\mathbf{v}}, \prod_{h} \zeta-\gamma_{h} \prod_{h} \zeta\right)\right| \leq\left|\left(\mathbf{v}-\overline{\mathbf{v}}, \prod_{h} \zeta-\zeta\right)\right|+\left|\left(\mathbf{v}-\overline{\mathbf{v}}, \zeta-\gamma_{h} \Pi_{h} \zeta\right)\right| \\
& \leq C h^{2}\|u\|_{1}\|\zeta\|_{1} \leq C h^{2}\left(\|\mathbf{p}\|_{1}+\|u\|_{1}+\int_{0}^{t}\|u\|_{1} d \tau\right)\|\zeta\|_{1}
\end{aligned}
$$

This completes the proof of the lemma. 
Lemma 3.3. Suppose that $b, c \in L^{\infty}\left(0, T ; W^{1, \infty}(\Omega)\right)$ and $u \in L^{\infty}\left(0, T ; H^{1}(\Omega)\right)$. Then for any $w_{h} \in W_{h}$ and $t \in(0, T]$, we have

$$
\begin{gathered}
\left|\left(b\left(P_{h} u-u\right), w_{h}\right)\right| \leq C h^{2}\|u\|_{1}\left\|w_{h}\right\|, \\
\left|\left(\int_{0}^{t} c\left(P_{h} u-u\right) d \tau, w_{h}\right)\right| \leq C h^{2} \int_{0}^{t}\|u\|_{1} d \tau\left\|w_{h}\right\| .
\end{gathered}
$$

Proof. The proof of (3.10) see lemma 4.3 in [15]. To prove (3.11), it follows (2.9) that

$$
\begin{aligned}
\left(\int_{0}^{t} c\left(P_{h} u-u\right) d \tau, w_{h}\right) & =\int_{0}^{t}\left(\left(c-P_{h} c\right)\left(P_{h} u-u\right), w_{h}\right) d \tau+\int_{0}^{t}\left(P_{h} u-u, w_{h} P_{h} c\right) d \tau \\
& \leq \int_{0}^{t}\left\|c-P_{h} c\right\|_{0, \infty}\left\|P_{h} u-u\right\|\left\|w_{h}\right\| d \tau \leq C h^{2} \int_{0}^{t}\|u\|_{1} d \tau\left\|w_{h}\right\| .
\end{aligned}
$$

This completes the proof of (3.11).

Lemma 3.4. Let $\mathbf{p} \in L^{\infty}\left(0, T ;\left(H^{1}(\Omega)\right)^{2}\right)$ and $u \in L^{\infty}\left(0, T ; H^{1}(\Omega)\right)$. Suppose that $(\tilde{\xi}, \tilde{\tau})$ satisfies the system (3.6) and the Assumptions 1 holds. Then any $t \in(0, T]$, we have

$$
\|\tilde{\tau}\| \leq C h \int_{0}^{t}(\|\tilde{\xi}\|+\|\operatorname{div} \tilde{\xi}\|) d \tau+C h^{2} \int_{0}^{t}\left(\|u\|_{1}+\|\mathbf{p}\|_{1}\right) d \tau .
$$

Proof. Given $\psi \in L^{2}(\Omega)$, let $\phi \in H^{2}(\Omega) \cap H_{0}^{1}(\Omega)$ be the solution of the adjoint problem

$$
\begin{array}{ll}
\text { (a) } \operatorname{div}(a \nabla \phi)=\psi, & x \in \Omega, ? \\
\text { (b) } \phi=0, & x \in \partial \Omega .
\end{array}
$$

Then the following elliptic regularity holds

$$
\|\phi\|_{2} \leq C\|\psi\| \text {. }
$$

By (2.4),(3.6) and $(\alpha \tilde{\xi}, a \nabla \phi)=-(\operatorname{div} \tilde{\xi}, \phi)$, we have

$$
\begin{aligned}
\left(\tilde{\tau}_{t}, \psi\right)= & \left(\tilde{\tau}_{t}, \operatorname{div}(a \nabla \phi)\right)=\left(\tilde{\tau}_{t}, \operatorname{div}(\Pi h(a \nabla \phi))\right) \\
= & -\left(\alpha \tilde{\xi},\left(I-\gamma_{h} \Pi_{h}\right) a \nabla \phi\right)-\left(\operatorname{div} \tilde{\xi}, \phi-P_{h} \phi\right)-\left(b \tilde{\tau}+\int_{0}^{t} c \tilde{\tau} d \tau, \operatorname{div}\left(\Pi_{h}(a \nabla \phi)\right)\right) \\
& +\left(\beta \tilde{\tau}+\int_{0}^{t} \gamma \tilde{\tau} d \tau, \gamma_{h}\left(\Pi_{h}(a \nabla \phi)\right)\right)-\left(\alpha \mathbf{p}+\beta u+\int_{0}^{t} \gamma u d \tau,\left(I-\gamma_{h}\right)\left(\Pi_{h}(a \nabla \phi)\right)\right) \\
& -\left(\operatorname{div} \Pi_{h}(a \nabla \phi), b\left(P_{h} u-u\right)+\int_{0}^{t} c\left(P_{h} u-u\right) d \tau\right) \\
& +\left(\beta\left(P_{h} u-u\right)+\int_{0}^{t} \gamma\left(P_{h} u-u\right) d \tau, \gamma_{h}\left(\Pi_{h}(a \nabla \phi)\right)\right) \\
= & H_{1}+H_{2}+H_{3}+H_{4}+H_{5}+H_{6}+H_{7} .
\end{aligned}
$$

Applying Lemma 2.5, Lemma 3.1-3.3 and (2.9), we obtain that

$$
\begin{aligned}
& \left|H_{1}\right| \leq C\|\tilde{\xi}\|\left\|\mid\left(I-\gamma_{h} \Pi_{h}\right) a \nabla \phi\right\| \leq C h\|\tilde{\xi}\|\|\phi\|_{2}, \\
& \left|H_{2}\right| \leq C h\left\|\operatorname { d i v } \tilde { \xi } \left|\|\mid \phi\|_{1} \leq C h\|\operatorname{div} \tilde{\xi}\|\|\phi\|_{2},\right.\right. \\
& \left|H_{3}\right| \leq C\left(\|\tilde{\tau}\|+\int_{0}^{t}\|\tilde{\tau}\| d \tau\right)\|\phi\|_{2}, \\
& \left|H_{4}\right| \leq C\left(\|\tilde{\tau}\|+\int_{0}^{t}\|\tilde{\tau}\| d \tau\right)|\phi|_{1} \leq C\left(\|\tilde{\tau}\|+\int_{0}^{t}\|\tilde{\tau}\| d \tau\right)\|\phi\|_{2}, \\
& \left|H_{5}\right| \leq C h^{2}\left(\|u\|_{1}+\|\mathbf{p}\|_{1}+\int_{0}^{t}\|u\|_{1} d \tau\right)\|\phi\|_{2}, \\
& \left|H_{6}\right| \leq C h^{2}\left(\|u\|_{1}+\int_{0}^{t}\|u\|_{1} d \tau\right)\|\phi\|_{2}, \\
& \left|H_{7}\right| \leq C h^{2}\left(\|u\|_{1}+\int_{0}^{t}\|u\|_{1} d \tau\right)\|\phi\|_{2} .
\end{aligned}
$$

It follows from the elliptic regularity (3.14) that

$\left|\left(\tilde{\tau}_{t}, \psi\right)\right| \leq C\left\{h(\|\tilde{\xi}\|+\|\operatorname{div} \tilde{\xi}\|)+\left(\|\tilde{\tau}\|+\int_{0}^{t}\|\tilde{\tau}\| d \tau\right)+h^{2}\left(\|u\|_{1}+\|\mathbf{p}\|_{1}+\int_{0}^{t}\|u\|_{1} d \tau \|\right)\right\}\|\psi\|$ so we get 


$$
\begin{aligned}
\left\|\left(\tilde{\tau}_{t}\right)\right\| & =\sup _{\psi \in L^{2}(\Omega) ; \psi \neq 0} \frac{\left(\tilde{\tau}_{t}, \psi\right)}{\|\psi\|} \\
& \leq C\left\{h(\|\tilde{\xi}\|+\|\operatorname{div} \tilde{\xi}\|)+h^{2}\left(\|u\|_{1}+\|\mathbf{p}\|_{1}+\int_{0}^{t}\|u\|_{1} d \tau\right)+\left(\|\tilde{\tau}\|+\int_{0}^{t}\|\tilde{\tau}\| d \tau\right)\right\}
\end{aligned}
$$

Since the following inequalities

$$
\|\tilde{\tau}\|=\left\|\int_{0}^{t} \tilde{\tau}_{t} d \tau\right\| \leq C \int_{0}^{t}\left\|\tilde{\tau}_{t}\right\| d \tau
$$

and

$$
\int_{0}^{t}\|\tilde{\tau}\| d \tau \leq C \int_{0}^{t}\left(\int_{0}^{\tau}\left\|\tilde{\tau}_{t}\right\| d s\right) d \tau \leq C \int_{0}^{t}\left(\int_{0}^{t}\left\|\tilde{\tau}_{t}\right\| d s\right) d \tau \leq C \int_{0}^{t}\left\|\tilde{\tau}_{t}\right\| d \tau
$$

hold, applying Gronwall's Lemma, we obtain that

$$
\begin{gathered}
\left\|\tilde{\tau}_{t}\right\| \leq C h(\|\tilde{\xi}\|+\|\operatorname{div} \tilde{\xi}\|)+C h^{2}\left(\|u\|_{1}+\|\mathbf{p}\|_{1}+\int_{0}^{t}\|u\|_{1} d \tau\right), \\
\|\tilde{\tau}\| \leq C h \int_{0}^{t}(\|\tilde{\xi}\|+\|\operatorname{div} \tilde{\xi}\|) \mathrm{d} \tau+C h^{2} \int_{0}^{t}\left(\|u\|_{1}+\|\mathbf{p}\|_{1}\right) d \tau
\end{gathered}
$$

This completes the proof of the lemma.

Theorem 3.5. Let $\mathbf{p} \in L^{\infty}\left(0, T ;\left(H^{1}(\Omega)\right)^{2}\right)$ and divp, $u, u_{t} \in L^{\infty}\left(0, T ; H^{1}(\Omega)\right)$. Suppose that $\left(\tilde{\mathbf{p}}_{h}, \tilde{u}_{h}\right)$ satisfies (3.1) and the Assumptions 1 holds. Then for any $t \in(0, T]$, the following error estimate

$$
\left\|u-\tilde{u}_{h}\right\| \leq C h\left\{\|u\|_{1}+\int_{0}^{t}\left(\|u\|_{1}+\|\mathbf{p}\|_{1}+\|\operatorname{div} \mathbf{p}\|_{1}\right) d \tau\right\}
$$

holds and for any $t \in(0, T]$, the following error estimates

$$
\begin{aligned}
& \left\|\mathbf{p}-\tilde{\mathbf{p}}_{h}\right\| \leq C h\left\{\|u\|_{1}+\|\mathbf{p}\|_{1}+\int_{0}^{t}\left(\|u\|_{1}+\|\mathbf{p}\|_{1}+\|\operatorname{div} \mathbf{p}\|_{1}\right) d \tau\right\} \\
& \left\|u_{t}-\tilde{u}_{h t}\right\| \leq C h\left\{\left\|u_{t}\right\|_{1}+\|u\|_{1}+\|\mathbf{p}\|_{1}+\|\operatorname{div} \mathbf{p}\|_{1}+\int_{0}^{t}\left(\|u\|_{1}+\|\mathbf{p}\|_{1}+\|\operatorname{div} \mathbf{p}\|_{1}\right) d \tau\right\}
\end{aligned}
$$

hold. Moreover, the following superconvergence results hold, for the variable $u$

$$
\begin{aligned}
& \left\|\tilde{u}_{h}-P_{h} u\right\| \leq C h^{2} \int_{0}^{t}\left(\|u\|_{1}+\|\mathbf{p}\|_{1}+\|\operatorname{div} \mathbf{p}\|_{1}\right) d \tau, \\
& \left\|\left(\tilde{u}_{h}-P_{h} u\right)_{t}\right\| \leq C h^{2}\left\{\|u\|_{1}+\|\mathbf{p}\|_{1}+\|\operatorname{div} \mathbf{p}\|_{1}+\int_{0}^{t}\left(\|u\|_{1}+\|\mathbf{p}\|_{1}+\|\operatorname{div} \mathbf{p}\|_{1}\right) d \tau\right\} .
\end{aligned}
$$

Proof. Let $\tilde{\sigma}=\tilde{\mathbf{p}}_{h}-\Pi_{h} \mathbf{p}$, then from $\tilde{\xi}=\tilde{\mathbf{p}}_{h}-\mathbf{p}=\tilde{\sigma}+\Pi_{h} \mathbf{p}-\mathbf{p}$ that (3.6) can be written as follows

(a) $\left(\alpha \tilde{\sigma}, \gamma_{h} \mathbf{v}_{h}\right)-\left(\operatorname{div} \mathbf{v}_{h}, \tilde{\tau}_{t}\right)-\left(b \tilde{\tau}+\int_{0}^{t} c \tilde{\tau} d \tau, \operatorname{div} \mathbf{v}_{h}\right)+\left(\beta \tilde{\tau}+\int_{0}^{t} \gamma \tilde{\tau} d \tau, \gamma_{h} \mathbf{v}_{h}\right)$

$$
\begin{aligned}
= & \left(\alpha\left(\mathbf{p}-\Pi_{h} \mathbf{p}\right), \gamma_{h} \mathbf{v}_{h}\right)+\left(\alpha \mathbf{p},\left(I-\gamma_{h}\right) \mathbf{v}_{h}\right)+\left(\beta u+\int_{0}^{t} \gamma u d \tau,\left(I-\gamma_{h}\right) \mathbf{v}_{h}\right) \\
& +\left(b\left(P_{h} u-u\right)+\int_{0}^{t} c\left(P_{h} u-u\right) d \tau, \operatorname{div} \mathbf{v}_{h}\right) \\
& -\left(\beta\left(P_{h} u-u\right)+\int_{0}^{t} \gamma\left(P_{h} u-u\right) d \tau, \gamma_{h} \mathbf{v}_{h}\right), \forall \mathbf{v}_{h} \in \mathbf{V}_{h}, t \in(0, T]
\end{aligned}
$$

(b) $\left(\operatorname{div} \tilde{\sigma}, w_{h}\right)=\left(\operatorname{div}\left(\mathbf{p}-\Pi_{h} \mathbf{p}\right), w_{h}\right)=0, \quad \forall w_{h} \in \mathbf{W}_{h}, t \in(0, T]$,

(c) $\tilde{\tau}(0)=0$.

Taking $w_{h}=\operatorname{div} \tilde{\sigma}$ in $(b)$ equation in (3.21), we obtain $\|\operatorname{div} \tilde{\sigma}\|=0$, which implies

$$
\operatorname{div} \tilde{\sigma}=\operatorname{div}\left(\tilde{\mathbf{p}}_{h}-\Pi_{h} \mathbf{p}\right)=0 .
$$

Taking $\mathbf{v}_{h}=\tilde{\sigma}$ in (a) equation in (3.21), we get

$$
\begin{aligned}
\left(\alpha \tilde{\sigma}, \gamma_{h} \tilde{\sigma}\right)= & -\left(\beta \tilde{\tau}+\int_{0}^{t} \gamma \tilde{\tau} d \tau, \gamma_{h} \tilde{\sigma}\right)+\left(\alpha\left(\mathbf{p}-\Pi_{h} \mathbf{p}\right), \gamma_{h} \tilde{\sigma}\right)+\left(\alpha \mathbf{p},\left(I-\gamma_{h}\right) \tilde{\sigma}\right) \\
& +\left(\beta u+\int_{0}^{t} \gamma u d \tau,\left(I-\gamma_{h}\right) \tilde{\sigma}\right)-\left(\beta\left(P_{h} u-u\right)+\int_{0}^{t} \gamma\left(P_{h} u-u\right) d \tau, \gamma_{h} \tilde{\sigma}\right)
\end{aligned}
$$

Similarly to the proofs of Lemma 2.4 and Lemma 3.3, we have 


$$
\left|\left(\beta u+\int_{0}^{t} \gamma u d \tau,\left(I-\gamma_{h}\right) \mathbf{v}_{h}\right)\right| \leq C h\left(\|u\|_{1}+\int_{0}^{t}\|u\|_{1} d \tau\right)\left\|\mathbf{v}_{h}\right\| .
$$

From Lemma 2.2, we get

$$
\left(\alpha \tilde{\sigma}, \gamma_{h} \tilde{\sigma}\right) \geq C_{1}^{-1}\|\tilde{\sigma}\|^{2} .
$$

Combining (3.23) with (3.24) and (3.25), and using (2.22), (2.23), (2.6), (2.9), we have

$$
\begin{aligned}
C_{1}^{-1}\|\tilde{\sigma}\|^{2} \leq & C\left(\|\tilde{\tau}\|+\int_{0}^{t}\|\tilde{\tau}\| d \tau\right)\left\|\gamma_{h} \tilde{\sigma}\right\|+C h\|\mathbf{p}\|_{1}\left\|\gamma_{h} \tilde{\sigma}\right\|+C h\|\mathbf{p}\|_{1}\|\tilde{\sigma}\| \\
& +C h\left(\|u\|_{1}+\int_{0}^{t}\|u\|_{1} d \tau\right)\|\tilde{\sigma}\|+C h\left(\|u\|_{1}+\int_{0}^{t}\|u\|_{1} d \tau\right)\left\|\gamma_{h} \tilde{\sigma}\right\|
\end{aligned}
$$

it follows from Lemma 2.1 that

$$
\|\tilde{\sigma}\|^{2} \leq C\left(\|\tilde{\tau}\|+\int_{0}^{t}\|\tilde{\tau}\| d \tau\right)+C h\left(\|\mathbf{p}\|_{1}+\|u\|_{1}+\int_{0}^{t}\|u\|_{1} d \tau\right)
$$

(3.26) together with (2.6), (2.7) and (3.22), we obtain

$\|\tilde{\xi}\| \leq\|\tilde{\sigma}\|+\left\|\Pi_{h} \mathbf{p}-\mathbf{p}\right\| \leq C\left(\|\tilde{\tau}\|+\int_{0}^{t}\|\tilde{\tau}\| d \tau\right)+C h\left(\|\mathbf{p}\|_{1}+\|u\|_{1}+\int_{0}^{t}\|u\|_{1} d \tau\right)$,

$\|\operatorname{div} \tilde{\xi}\| \leq\|\operatorname{div} \tilde{\sigma}\|+\left\|\operatorname{div}\left(\Pi_{h} \mathbf{p}-\mathbf{p}\right)\right\| \leq C h\|\operatorname{div} \mathbf{p}\|_{1}$.

Substituting (3.27) and (3.28) back into (3.12) yields

$$
\begin{aligned}
\|\tilde{\tau}\| \leq & C h \int_{0}^{t}\left(\|\tilde{\tau}\|+\int_{0}^{\tau}\|\tilde{\tau}\| d s\right) d \tau+C h^{2} \int_{0}^{t}\left(\|u\|_{1}+\|\mathbf{p}\|_{1}+\int_{0}^{\tau}\|u\|_{1} d s\right) d \tau \\
& +C h^{2} \int_{0}^{t}\|\operatorname{div} \mathbf{p}\|_{1} d \tau+C h^{2} \int_{0}^{t}\left(\|u\|_{1}+\|\mathbf{p}\|_{1}\right) d \tau \\
\leq & C h \int_{0}^{t}\|\tilde{\tau}\| d \tau+C h^{2} \int_{0}^{t}\left(\|u\|_{1}+\|\mathbf{p}\|_{1}+\|\operatorname{div} \mathbf{p}\|_{1}\right) d \tau
\end{aligned}
$$

Applying Grownwall's Lemma we have

$$
\|\tilde{\tau}\| \leq C h^{2} \int_{0}^{t}\left(\|u\|_{1}+\|\mathbf{p}\|_{1}+\|\operatorname{div} \mathbf{p}\|_{1}\right) d \tau
$$

This completes the proof of (3.19).

Combining (2.9) with (3.29) we obtain (3.16). Substituting (3.29) back into (3.27) yields

$$
\|\tilde{\xi}\| \leq C h\left\{\|u\|_{1}+\|\mathbf{p}\|_{1}+\int_{0}^{t}\left(\|\mathbf{p}\|_{1}+\|u\|_{1}+\|\operatorname{div} \mathbf{p}\|_{1}\right) d \tau\right\}
$$

Combining (3.28) with (3.30), we get (3.17). From (3.15) and (3.28), (3.30), we obtain

$$
\left\|\tilde{\tau}_{t}\right\| \leq C h^{2}\left\{\|u\|_{1}+\|\mathbf{p}\|_{1}+\|\operatorname{div} \mathbf{p}\|_{1}+\int_{0}^{t}\left(\|\mathbf{p}\|_{1}+\|u\|_{1}+\|\operatorname{div} \mathbf{p}\|_{1}\right) d \tau\right\} .
$$

This completes the proof of (3.20).

From (3.20) and (2.9) we get (3.18) directly. This completes the proof of the theorem.

\section{The Error Estimates}

In this section, we will use the properties of the mixed covolume elliptic projection $\left\{\tilde{\mathbf{p}}_{h}, \tilde{u}_{h}\right\}$ to derive the optimal rate of convergence for the approximate velocity and pressure in the $\mathbf{H}(\operatorname{div} ; \Omega)$-norm and $L^{2}$-norm.

In order to get our main results, we need the following lemmas.

Lemma 4.1 If $v \in L^{\infty}\left(0, T ; L^{\infty}(\Omega)\right)$. Then for any $\mathbf{v}_{h} \in \mathbf{V}_{h}, u_{h} \in W_{h}$ we have

$$
\left|B\left(\gamma_{h} \mathbf{v}_{h}, v u_{h}\right)\right| \leq C\|v\|_{0, \infty}\left\|u _ { h } \left|\left\|\mid \gamma_{h} \mathbf{v}_{h}\right\|\right.\right. \text {. }
$$

Proof. By the definition of the bilinear $B$, we have

$$
B\left(\gamma_{h} \mathbf{v}_{h}, v u_{h}\right)=\sum_{i=1}^{N_{s}}\left(\int_{\partial K_{p_{i}}^{*} \cap K_{P_{i},}} v u_{h} \gamma_{h} \mathbf{v}_{h} \cdot \mathbf{n}_{i} d s+\int_{\partial K_{P_{i}}^{*} \cap K_{P_{i} R}} v u_{h} \gamma_{h} \mathbf{v}_{h} \cdot \mathbf{n}_{i} d s\right),
$$

where $\mathbf{n}_{i}$ stands for the unite outer normal direction of $K_{P_{i}}^{*}, i=1, \cdots, N_{s}$.

For any $i=1$, see Fig. $1, K_{P_{1}}^{*} \cap K_{P_{1} L}=\Delta A_{1} B_{1} A_{3}$, with the mid-points $P_{1}$ of the side $A_{1} A_{3}$. 
Then $\partial K_{P_{1}}^{*} \cap K_{P_{1} L}=\overline{A_{1} B_{1}}+\overline{B_{1} A_{3}}$. So that

$$
\int_{\partial K_{\text {म }}^{*} \cap K_{K_{L}}}\left|u_{h} \gamma_{h} \mathbf{v}_{h} \cdot \mathbf{n}_{1}\right| d s=\int_{\overline{A_{1} B_{1}}}\left|u_{h} \gamma_{h} \mathbf{v}_{h} \cdot \mathbf{n}_{11}\right| d s+\int_{\overline{B_{1} A_{3}}}\left|u_{h} \gamma_{h} \mathbf{v}_{h} \cdot \mathbf{n}_{12}\right| d s,
$$

where $\mathbf{n}_{11}$ is the unite outer normal vector to the edge $A_{1} B_{1}, \mathbf{n}_{12}$ is the unite outer normal vector to the edge $B_{1} A_{3}$.

Let $K$ be an element with $e$ as an edge. It is well known (see [2]) that there exists a constant $C$ such that for any function $w \in H^{1}(K),\|w\|_{e} \leq C\left(h_{e}^{-\frac{1}{2}}\|w\|_{0, K}+h_{e}^{\frac{1}{2}}|w|_{1, K}\right)$, where $h_{e}$ is the length of the edge $e$ and $C$ depends only on the minimum angle of $K$.

Noting that $u_{h} \gamma_{h} \mathbf{v}_{h} \cdot \mathbf{n}_{11}$ is a constant in $\triangle A_{1} B_{1} A_{3}$, we get

$$
\int_{\overline{A_{1} B_{1}}}\left|u_{h} \gamma_{h} \mathbf{v}_{h} \cdot \mathbf{n}_{11}\right| d s \leq C h^{\frac{1}{2}}\left\|u_{h} \gamma_{h} \mathbf{v}_{h} \cdot \mathbf{n}_{11}\right\|_{\overline{A_{1} B_{1}}} \leq C\left\|u_{h} \gamma_{h} \mathbf{v}_{h} \cdot \mathbf{n}_{11}\right\|_{0, \Delta A_{1} B_{1} A_{3}}
$$

Similarly, we get

so we have

$$
\int_{\overline{B_{1} A_{3}}}\left|u_{h} \gamma_{h} \mathbf{v}_{h} \cdot \mathbf{n}_{12}\right| d s \leq C\left\|u_{h} \gamma_{h} \mathbf{v}_{h} \cdot \mathbf{n}_{12}\right\|_{0, \Delta A_{1} B_{1} A_{3}}
$$

$$
\begin{aligned}
\int_{\partial K_{P^{*}}^{*} \cap K_{K_{1} L}}\left|u_{h} \gamma_{h} \mathbf{v}_{h} \cdot \mathbf{n}_{1}\right| d s & \leq C\left(\left\|u_{h} \gamma_{h} \mathbf{v}_{h} \cdot \mathbf{n}_{11}\right\|_{0, \Delta A_{1} B_{1} A_{3}}+\left\|u_{h} \gamma_{h} \mathbf{v}_{h} \cdot \mathbf{n}_{12}\right\|_{0, \Delta A_{1} B_{1} A_{3}}\right) \\
& \leq C\left\|u_{h}\right\|_{0, K_{P}^{*}}\left\|\gamma_{h} \mathbf{v}_{h}\right\|_{0, K_{R_{1}}^{*}}
\end{aligned} .
$$

Similarly, for $i=2, \cdots, N_{s}$, we derive that

$$
\int_{\partial K_{P_{i}}^{*} \cap K_{P_{i} L}}\left|u_{h} \gamma_{h} \mathbf{v}_{h} \cdot \mathbf{n}_{i}\right| d s \leq C\left\|u_{h}\right\|_{0, K_{P_{i}}^{*}}\left\|\gamma_{h} \mathbf{v}_{h}\right\|_{0, K_{P_{i}}^{*}}
$$

and likewise for $i=1, \cdots, N \mathrm{~s}$, we have

Hence we obtain

$$
\left|B\left(\gamma_{h} \mathbf{v}_{h}, v u_{h}\right)\right| \leq C\|v\|_{0, \infty} \sum_{i=1}^{N_{s}} C\left\|u_{h}\right\|_{0, K_{P_{i}^{*}}^{*}}\left\|\gamma_{h} \mathbf{v}_{h}\right\|_{0, K_{P_{i}^{*}}^{*}} \leq C\|v\|_{0, \infty}\left\|u_{h}\right\|\left\|\gamma_{h} \mathbf{v}_{h}\right\|
$$

This completes the proof of the lemma.

Lemma 4.2 If $b, c \in L^{\infty}\left(0, T ; W^{1, \infty}(\Omega)\right)$. Then for any $u_{h} \in W_{h}, \mathbf{v}_{h} \in \mathbf{V}_{h}$ and $t \in(0, T]$, we have

$$
\left|B\left(\gamma_{h} \mathbf{v}_{h},\left(b-P_{h} b\right) u_{h}+\int_{0}^{t}\left(c-P_{h} c\right) u_{h} d \tau\right)\right| \leq C h\left(\left\|u_{h}\right\|+\int_{0}^{t}\left\|u_{h}\right\| d \tau\right)\left\|\gamma_{h} \mathbf{v}_{h}\right\| \text {. }
$$

Proof. Taking $v=b-P_{h} b$ in Lemma 4.1, by the property of operator $P_{h}$, we obtain

$$
\|v\|_{0, \infty}=\left\|b-P_{h} b\right\|_{0, \infty} \leq C h|b|_{1, \infty} \leq C h\|b\|_{L^{\infty}\left(0, T ; W^{1, \infty}(\Omega)\right)} \leq C h
$$

which implies

$$
\left|B\left(\gamma_{h} \mathbf{v}_{h},\left(b-P_{h} b\right) u_{h}\right)\right| \leq C h\left\|u_{h}|||| \gamma_{h} \mathbf{v}_{h}\right\| \text {. }
$$

Similarly, taking $v u_{h}=\int_{0}^{t}\left(c-P_{h} c\right) u_{h} d \tau$ in Lemma 4.1, we have

$$
\left|B\left(\gamma_{h} \mathbf{v}_{h}, \int_{0}^{t}\left(c-P_{h} c\right) u_{h} d \tau\right)\right| \leq C h\|c\|_{L^{\infty}\left(0, T ; W^{1, \infty}(\Omega)\right)} \int_{0}^{t}\left\|u_{h}\right\| d \tau\left\|\gamma_{h} \mathbf{v}_{h}\right\| \leq C h \int_{0}^{t}\left\|u_{h}\right\| d \tau\left\|\gamma_{h} \mathbf{v}_{h}\right\| .
$$

Using the triangle inequality now completes the proof.

Subtracting (2.2) from (2.30), and considering the mixed covolume elliptic projection introduced in (3.1), we obtain the error equations 
(a) $\left(\alpha\left(\mathbf{p}_{h}-\tilde{\mathbf{p}}_{h}\right)+\beta\left(u_{h}-\tilde{u}_{h}\right)+\int_{0}^{t} \gamma\left(u_{h}-\tilde{u}_{h}\right) d \tau, \gamma_{h} \mathbf{v}_{h}\right)$

$$
\begin{array}{ll}
-\left(\operatorname{div} \mathbf{v}_{h},\left(u_{h t}-\tilde{u}_{h t}\right)+\left(\left(P_{h} b\right) u_{h}-b \tilde{u}_{h}\right)+\int_{0}^{t}\left(\left(P_{h} c\right) u_{h}-c \tilde{u}_{h} d \tau\right)\right. \\
+B\left(\gamma_{h} \mathbf{v}_{h},\left(b-P_{h} b\right) u_{h}+\int_{0}^{t}\left(c-P_{h} c\right) u_{h} d \tau\right)=0, & \forall \mathbf{v}_{h} \in \mathbf{V}_{h}, t \in(0, T], \\
\text { (b) } \quad\left(u_{h t}-u_{t}, w_{h}\right)+\left(\operatorname{div}\left(\mathbf{p}_{h}-\tilde{\mathbf{p}}_{h}\right), w_{h}\right)=0, & \forall w_{h} \in \mathbf{W}_{h}, t \in(0, T], \\
\text { (c) } \quad\left(u_{h}(0)-\tilde{u}_{h}(0), w_{h}\right)=0, & \forall w_{h} \in \mathbf{W}_{h} .
\end{array}
$$

Let $u_{h}-u=u_{h}-\tilde{u}_{h}+\tilde{u}_{h}-u=\eta+\tilde{\eta}, \mathbf{p}_{h}-\mathbf{p}=\mathbf{p}_{h}-\tilde{\mathbf{p}}_{h}+\tilde{\mathbf{p}}_{h}-\mathbf{p}=\xi+\tilde{\xi}$. Then (4.1) can be rewritten as

(a) $\left(\alpha \xi+\beta \eta+\int_{0}^{t} \gamma \eta d \tau, \gamma_{h} \mathbf{v}_{h}\right)-\left(\operatorname{div} \mathbf{v}_{h}, \eta_{t}+\left(P_{h} b\right) \eta+\int_{0}^{t}\left(P_{h} c\right) \eta d \tau\right)$

$$
\begin{array}{ll}
\left.-\left(\operatorname{div} \mathbf{v}_{h},\left(P_{h} b-b\right) \tilde{u}_{h}\right)+\int_{0}^{t}\left(c-P_{h} c\right) \tilde{u}_{h} d \tau\right)+B\left(\gamma_{h} \mathbf{v}_{h},\left(b-P_{h} b\right) \eta+\int_{0}^{t}\left(c-P_{h} c\right) \eta d \tau\right) \\
+B\left(\gamma_{h} \mathbf{v}_{h},\left(b-P_{h} b\right) \tilde{u}_{h}+\int_{0}^{t}\left(c-P_{h} c\right) \tilde{u}_{h} d \tau\right)=0, & \forall \mathbf{v}_{h} \in \mathbf{V}_{h}, t \in(0, T], \\
\text { (b) } \quad\left(\eta_{t}, w_{h}\right)+\left(\operatorname{div} \xi, w_{h}\right)=-\left(\tilde{\eta}_{t}, w_{h}\right), & \forall w_{h} \in \mathbf{W}_{h}, t \in(0, T], \\
\text { (c) } \eta_{h}(0)=0, \quad\left(u_{h t}-\tilde{u}_{h t}\right) & \forall w_{h} \in \mathbf{W}_{h} .
\end{array}
$$

Taking $w_{h}=\operatorname{div} \xi$ in equation $(b)$ in (4.2), we get

$\|\operatorname{div} \xi\| \leq\left\|\eta_{t}\right\|+\left\|\tilde{\eta}_{t}\right\|$

Taking $\mathbf{v}_{h}=\xi$ in equation $(a)$ and $w_{h}=\eta_{t}+\left(P_{h} b\right) \eta$ in equation (b) in (4.2), we have

$$
\begin{aligned}
\left(\alpha \xi, \gamma_{h} \xi\right)+\left(\eta_{t}, \eta_{t}\right)=- & \left.\left(\beta \eta+\int_{0}^{t} \gamma \eta d \tau, \gamma_{h} \xi\right)+\left(\operatorname{div} \xi,\left(P_{h} b-b\right) \tilde{u}_{h}\right)+\int_{0}^{t}\left(P_{h} c-c\right) \tilde{u}_{h} d \tau\right) \\
& +\left(\operatorname{div} \xi, \int_{0}^{t}\left(P_{h} c\right) \eta d \tau\right)-B\left(\gamma_{h} \xi,\left(b-P_{h} b\right) \eta+\int_{0}^{t}\left(c-P_{h} c\right) \eta d \tau\right) \\
& -B\left(\gamma_{h} \xi,\left(b-P_{h} b\right) \tilde{u}_{h}+\int_{0}^{t}\left(c-P_{h} c\right) \tilde{u}_{h} d \tau\right)-\left(\eta_{t},\left(P_{h} b\right) \eta\right)-\left(\tilde{\eta}_{t}, \eta_{t}+\left(P_{h} b\right) \eta\right) .
\end{aligned}
$$

Using (3.16), we obtain

$$
\begin{aligned}
&\left\|\tilde{u}_{h}\right\| \leq\left\|\tilde{u}_{h}-u\right\|+\|u\| \leq C h\left(\|u\|_{1}+\int_{0}^{t}\left(\|u\|_{1}+\|\mathbf{p}\|_{1}+\|\operatorname{div} \mathbf{p}\|_{1}\right) d \tau+\|u\|\right. \\
& \leq C\left(\|u\|_{1}+\int_{0}^{t}\left(\|u\|_{1}+\|\mathbf{p}\|_{1}+\|\operatorname{div} \mathbf{p}\|_{1}\right) d \tau\right.
\end{aligned}
$$

which gives

$$
\begin{aligned}
\left\|\left(P_{h} b-b\right) \tilde{u}_{h}+\int_{0}^{t}\left(P_{h} c-c\right) \tilde{u}_{h} d \tau\right\| & \leq C h\left(\|b\|_{1, \infty}\left\|\tilde{u}_{h}\right\|+\int_{0}^{t}\|c\|_{1, \infty}\left\|\tilde{u}_{h}\right\| d \tau\right) \\
& \leq C h\left(\|u\|_{1}+\int_{0}^{t}\left(\|u\|_{1}+\|\mathbf{p}\|_{1}+\|\operatorname{div} \mathbf{p}\|_{1}\right) d \tau\right.
\end{aligned} .
$$

Applying Lemma 4.2, we obtain

$\left|B\left(\gamma_{h} \xi,\left(b-P_{h} b\right) \eta+\int_{0}^{t}\left(c-P_{h} c\right) \eta d \tau\right)\right| \leq C h\left(\|\eta\|+\int_{0}^{t}\|\eta\| d \tau\right)\left\|\gamma_{h} \xi\right\|$,

$\mid B\left(\gamma_{h} \xi,\left(b-P_{h} b\right) \tilde{u}_{h}+\int_{0}^{t}\left(c-P_{h} c\right) \tilde{u}_{h} d \tau \mid \leq C h\left(\|u\|_{1}+\int_{0}^{t}\left(\|u\|_{1}+\|\mathbf{p}\|_{1}+\|\operatorname{div} \mathbf{p}\|_{1}\right) d \tau\right)\left\|\gamma_{h} \xi\right\|\right.$.

It follows from Lemma 2.4 and (4.3), (4.4) that

$$
\begin{aligned}
C_{1}^{-1} & \|\xi\|^{2}+\left\|\eta_{t}\right\|^{2} \\
\leq & \left(\alpha \xi, \gamma_{h} \xi\right)+\left(\eta_{t}, \eta_{t}\right) \\
\leq & C\left(\|\eta\|+\int_{0}^{t}\|\eta\| d \tau\right)^{2}+C\left(\int_{0}^{t}\|\eta\| d \tau\right)^{2}+C h^{2}\left(\|u\|_{1}+\int_{0}^{t}\left(\|u\|_{1}+\|\mathbf{p}\|_{1}+\|\operatorname{div} \mathbf{p}\|_{1}\right) d \tau\right)^{2}+C\left\|\tilde{\eta}_{t}\right\|^{2} \\
& +C h^{2}\left(\|\eta\|+\int_{0}^{t}\|\eta\| d \tau\right)^{2}+\frac{1}{2 C_{1}}\left\|\gamma_{h} \xi\right\|^{2}+\frac{1}{2}\left\|\eta_{t}\right\|^{2}+C\|\eta\|^{2}
\end{aligned}
$$

Furthermore, we get 


$$
\begin{aligned}
& \|\xi\|+\left\|\eta_{t}\right\| \\
& \leq C\left(\|\eta\|+\int_{0}^{t}\|\eta\| d \tau+\left\|\tilde{\eta}_{t}\right\|\right)+C h\left(\|\eta\|+\int_{0}^{t}\|\eta\| d \tau\right)+C h\left(\|u\|_{1}+\int_{0}^{t}\left(\|u\|_{1}+\|\mathbf{p}\|_{1}+\|\operatorname{div} \mathbf{p}\|_{1}\right) d \tau\right) \\
& \leq C\left\|\tilde{\eta}_{t}\right\|+C(1+h) \int_{0}^{t}\left\|\eta_{t}\right\| d \tau+C h\left(\|u\|_{1}+\int_{0}^{t}\left(\|u\|_{1}+\|\mathbf{p}\|_{1}+\|\operatorname{div} \mathbf{p}\|_{1}\right) d \tau\right) \\
& \leq C\left\|\tilde{\eta}_{t}\right\|+C \int_{0}^{t}\left\|\eta_{t}\right\| d \tau+C h\left(\|u\|_{1}+\int_{0}^{t}\left(\|u\|_{1}+\|\mathbf{p}\|_{1}+\|\operatorname{div} \mathbf{p}\|_{1}\right) d \tau\right)
\end{aligned}
$$

Applying Grownwall's Lemma and (3.18), we get

$$
\|\xi\|+\left\|\eta_{t}\right\| \leq C h\left\{\left\|u_{t}\right\|_{1}+\|u\|_{1}+\|\mathbf{p}\|_{1}+\|\operatorname{div} \mathbf{p}\|_{1}+\int_{0}^{t}\left(\|u\|_{1}+\|\mathbf{p}\|_{1}+\|\operatorname{div} \mathbf{p}\|_{1}\right) d \tau\right\}
$$

and

$$
\|\eta\| \leq C \int_{0}^{t}\left\|\eta_{t}\right\| d \tau \leq C h \int_{0}^{t}\left(\left\|u_{t}\right\|_{1}+\|u\|_{1}+\|\mathbf{p}\|_{1}+\|\operatorname{div} \mathbf{p}\|_{1}\right) d \tau
$$

Combining (4.3) with (3.18) and the estimate (4.5) of $\left\|\eta_{t}\right\|$, we obtain

$$
\|\operatorname{div} \xi\| \leq C h\left\{\left\|u_{t}\right\|_{1}+\|u\|_{1}+\|\mathbf{p}\|_{1}+\|\operatorname{div} \mathbf{p}\|_{1}+\int_{0}^{t}\left(\|u\|_{1}+\|\mathbf{p}\|_{1}+\|\operatorname{div} \mathbf{p}\|_{1}\right) d \tau\right\} .
$$

Using (4.5)-(4.7) and Theorem 3.5, we obtain the following main results.

Theorem 4.1 Let $(\mathbf{p}, u)$ and $\left(\mathbf{p}_{h}, u_{h}\right)$ be the solution of (2.2) and (2.30), respectively, and suppose that $\mathbf{p} \in L^{\infty}\left(0, T ;\left(H^{1}(\Omega)\right)^{2}\right), \operatorname{divp}, u, u_{t} \in L^{\infty}\left(0, T ; H^{1}(\Omega)\right)$ and the Assumptions 1 holds. Then for any $t \in[0, T]$, the following error estimate holds

$$
\left\|u-u_{h}\right\| \leq C h\left\{\|u\|_{1}+\int_{0}^{t}\left(\left\|u_{t}\right\|_{1}+\|u\|_{1}+\|\mathbf{p}\|_{1}+\|\operatorname{div} \mathbf{p}\|_{1}\right) d \tau\right\}
$$

and for any $t \in(0, T]$, the following error estimates hold

$$
\begin{aligned}
& \left\|u_{t}-u_{h t}\right\| \leq C h\left\{\left\|u_{t}\right\|_{1}+\|u\|_{1}+\|\mathbf{p}\|_{1}+\|\operatorname{div} \mathbf{p}\|_{1}+\int_{0}^{t}\left(\|u\|_{1}+\|\mathbf{p}\|_{1}+\|\operatorname{div} \mathbf{p}\|_{1}\right) d \tau\right\}, \\
& \left\|\mathbf{p}-\mathbf{p}_{h}\right\| \leq C h\left\{\left\|u_{t}\right\|_{1}+\|u\|_{1}+\|\mathbf{p}\|_{1}+\|\operatorname{div} \mathbf{p}\|_{1}+\int_{0}^{t}\left(\|u\|_{1}+\|\mathbf{p}\|_{1}+\|\operatorname{div} \mathbf{p}\|_{1}\right) d \tau\right\}
\end{aligned}
$$

\section{References}

[1] R. Adams, Sobolev spaces. Academic Press, New York(1975).

[2] D. Arnold, An interior penalty finite element method with discontinuous elements. SIAM J Numer Anal 19, 742-760(1982).

[3] H. Che, Error estimates for mixed element methods for for pseudo-parabolic integro-differential equations. Chinese J Engineering Mathematics 26(6), 1033-1039.

[4] S. H. Chou, D. Y. Kwak, and P. S. Vassilevski, Mixed covolume methods for the elliptic problems on triangular grids. SIAM J Numer Anal 35, 1850-1861(1998).

[5] P. G. Ciarlet, The finite element method for elliptic problems. North-Holland, Amsterdam(1978).

[6] S. Cui, The global solutions for a class of nonlinear integro-differential equations. ACTA Mathematicae Applicatae Sinica 16(2), 191-200(1993).

[7] X. Cui, Sobolev-Volterra projection and numerical analysis of finite element methods for integro-differential equations. ACTA Mathematicae Applicatae Sinica 24(3), 441-455(2001).

[8] M. Fortin, An analysis of the convergence of mixed finite element methods. RAIRO Anal. Numer. 11, 341-354(1977).

[9] Z. Jiang, $L^{\infty}\left(L^{2}\right)$ and $L^{\infty}\left(L^{\infty}\right)$ error estimates for mixed methods for integro-differential equations of parabolic type. Math. Model. Numer. Anal. 33, 531-546(1999).

[10] B. Liu, H. Song, Solution to mixed problem for some nonlinear pseudo-parabolic partial equations. J. Information Engineering University 6(2), 35-38(2005).

[11] M. Ptashnyk, Degenerate quasilinear pseudoparabolic equations with memory terms and variational inequalities. Nonlinear Analysis: Theory, Methods and Applications 66(12), 2653-2675(2007). 
[12] P. A. Raviart, J. M. Thomas, A mixed finite element methods for second order elliptic problems. Lecture Notes in Mathematical 606, 292-315, Springer, Berlin, Heidelberg(1977).

[13] T. F. Russell, Rigorous block-centered discretizations on irregular grids: improved simulation of complex reservoir systems, Technical Report No. 3, Project Report, Reservoir Simulation Research Corporation, 1995.

[14] S. Wang, Initial boundary value problem for the semilinear integro-differential equations of pseudo-hyperbolic type. ACTA Mathematicae Applicatae Sinica 18(4), 576-578(1995).

[15] S. Yang, Z. Jiang, Mixed covolume method for parabolic problems on triangular grids. Applied Mathematics and Computation 215, 1251-1265(2009).

[16] Z. Zhou, H. Chen, H1-Galerkin mixed finite element method for pseudo-parabolic integrodifferential equation. J. Shandong Normal University(Natural Science) 20(2), 3-7(2005). 